\title{
Probabilistic Analysis of Abnormal Behaviour Detection in Activities of Daily Living
}

\author{
M. Garcia-Constantino*, A. Konios ${ }^{\dagger}$, I. Ekerete*, S. Christopoulos ${ }^{\dagger}$, \\ C. Shewell*, C. Nugent* and G. Morrison ${ }^{\S}$ \\ ${ }^{*}$ School of Computing, Ulster University, Jordanstown, United Kingdom. \\ ${ }^{\dagger}$ Institute for Future Transport and Cities, Coventry University, Coventry, United Kingdom. \\ ${ }^{\ddagger}$ The Lava Group, Belfast, United Kingdom.
}

\begin{abstract}
This paper presents a probabilistic approach for the detection of abnormal behaviour in Activities of Daily Living (ADLs) from sensor data collected from 30 participants. The ADLs considered are: (i) preparing and drinking tea, and (ii) preparing and drinking coffee. Abnormal behaviour detected in the context of these activities can be an indicator of a progressive health problem or the occurrence of a hazardous incident. The approach presented considers the temporal aspect of the sequences of actions that are part of each ADL and that vary between participants. The average and standard deviation for the durations of each action were calculated to define an average time and a range in which a behaviour could be considered as normal for each stage and activity. The Cumulative Distribution Function (CDF) was used to obtain the probabilities of abnormal behaviours related to the early and late completion of activities and stages within an activity. The data analysis show that CDF can provide accurate and reliable results regarding the presence of abnormal behaviour in stages and activities that last over a minute. The approach presented could be used to train and improve machine learning algorithms of abnormal behaviour detection.
\end{abstract}

Index Terms-Activities of Daily Living, ADLs, Cumulative Distribution Function, CDF, Probabilistic Analysis

\section{INTRODUCTION}

In general, the detection of abnormal behaviour in Activities of Daily Living (ADLs) can be an indicator of a progressive health problem taking place (dementia, osteoporosis, arthritis, etc.) or the occurrence of a hazardous incident (falls, burns, cuts, food or smoke intoxication, etc.). Monitoring ADLs performed by elderly people for detecting abnormal behaviour is of particular importance due to the potential life changing consequences that could result from not acting timely. Using sensors that can be placed within the environment and in appliances of interest, as opposed to wearable sensors, has the main advantage of not being intrusive for the users.

This work continues the research presented in [5], which considered the use of Petri nets to model and verify ADLs. In this case, a probabilistic approach is applied to sensor data collected from participants for the ADL of "preparing and drinking a hot beverage" with the variants of tea or

ACKNOWLEDGEMENT: Invest Northern Ireland is acknowledged for supporting this project under the Competence Centre Programs Grant RD0513853 - Connected Health Innovation Centre. coffee. In addition of considering the completion or noncompletion of an activity as a means to identify normal or abnormal behaviour, the research presented considers: (i) the sequence of actions (steps) followed by the participants, (ii) the duration of actions that are part of an activity, and (iii) the total duration of an activity. While "preparing and drinking a hot beverage" with two choices of drink type is a relatively simple and common ADL to perform, considering the sequences of actions and the durations for different users provides an interesting insight and granularity from which abnormal behaviours can be detected.

In this case, it is assumed that a user completes an activity regardless of the number and sequence of steps involved, and of the time taken for the completion. Particularly, it is the information about the temporal aspects of the activities (i.e. duration) that is used to find patterns that could support in abnormal behaviour detection. If there are patterns found from sensor data outside the norm for an ADL that a person within a certain age range performs, then it could be inferred that the person has a health problem and must be attended by carers. In addition, the sensor system could send prompts to the user suggesting the completion of an activity. Detection of active appliances which could represent a hazard when unattended (e.g. stove or oven) could trigger alert messages sent to the user, relatives and/or carers.

The main contribution of this paper is the probabilistic analysis of the sequence of actions performed by 30 volunteers for the detection of patterns that could indicate abnormal behaviours. Furthermore, the dataset collected includes data from a number of sensors (contact, thermal and accelerometer) for each user, which provides a granularity for the analysis of the data from different perspectives. In this paper, only the contact sensor data is considered, as it provides accurate and appropriate data of users' interactions with appliances and resources involved in the ADL for the type of analysis presented. The thermal and accelerometer data will be used in future work.

The remainder of the paper is organised as follows: Section II presents the related work in the areas of activity recognition and probabilistic analysis of intelligent systems. Section III describes the environment setup considered for the data collection of ADLs. Section IV describes the participants and 
scenarios considered. Section V presents the data analysis and the evaluation of the results. Finally, Section VI presents the conclusions.

\section{BACKGROUND}

One of the ways in which activity recognition can be classified is in terms of vision-based versus sensor-based [3]. While the former can provide more information to an activity recognition system, it has the disadvantage of not protecting the privacy of the users. Activity recognition using sensors is typically classified in terms of wearable sensors versus dense sensors. Wearable sensors can be worn by users in parts of their body or in their clothes [4], [6], [16]. While wearable sensors have the advantage of monitoring and collecting data regardless of the location of the users, some of the main disadvantages are that the users are responsible for their correct use and for charging the battery. Dense sensors are attached to objects in the environment with which the user interacts (e.g. kitchen, bathroom, kettle) [15]. These sensors have the advantage of not being intrusive and typically do not require the user to charge them periodically. However, the main disadvantages of dense sensors are that they need to be well placed in the environment and within an adequate setting.

The use of sensors for activity recognition in ADLs to detect and predict abnormal behaviour has been investigated in the literature. A wireless sensor network to monitor and detect behaviour of eldery people is presented in [14]. The system generates sensor activity patterns which are used to predict abnormal behaviour based on a classification model of regular and irregular sensor activity. In [9] a sensor network to monitor elderly people's behaviour and to identify and prevent abnormal behaviour using neural networks is introduced. The results presented in [9] suggest that duration of activities is the most effective way to represent and handle a large dataset of sensor data.

In [10], occupancy data represented as temporal sequences of activities for identifying and predicting behaviours is investigated. The sensors used in [10] to detect occupancy are Passive Infra-red Sensors (PIR), door/window entry point sensors and bed/sofa pressure sensors. An automated method based on Markov Chains Model (MCM) to classify abnormal sequences of activities is presented in [17]. The probability distribution of the spatiotemporal activity data of an elderly user over 128 days is analysed. Another probabilistic approach is presented in [1], in which data from motion, door and pressure sensors is collected from 40 households and presented in an abstract layer. A probabilistic spatio-temporal model is then produced to present a summary of daily behaviour where significant changes are defined as anomalies.

The Online Daily Habit Model and Anomaly Detection (ODHMAD) model [11] is comprised by an Online Activity Recognition (OAR) model and a Dynamic Daily Habit
Modeling (DDHM) component. The ODHMAD model aims to present real-time personalised ADL recognition, habit modeling and anomaly detection for elderly people. In [5], Petri nets are used to model three ADLs for the identification of the completion or non-completion of the activities as indicators of normal or abnormal behaviour, respectively. The description of the sensor system considered and the developed Petri net model and its verification are presented. The work presented in [7] introduces a unification approach to help in the detection of normal and abnormal behaviour based on the performance of ADLs by eldery people in care homes equipped with different types of sensors.

\section{ENVIRONMENT SETUP}

The ADLs considered for the data collection and analysis are: (i) preparing and drinking tea, and (ii) preparing and drinking coffee. These ADLs are usually carried out in the kitchen and are based on the ones used in [2], where they were modelled using ontologies. The environment in which the data for the kitchen ADLs was collected is the one at the smart kitchen in the Smart Environments Research Group (SERG) ${ }^{1}$ at Ulster University (see layout in Fig. 1).

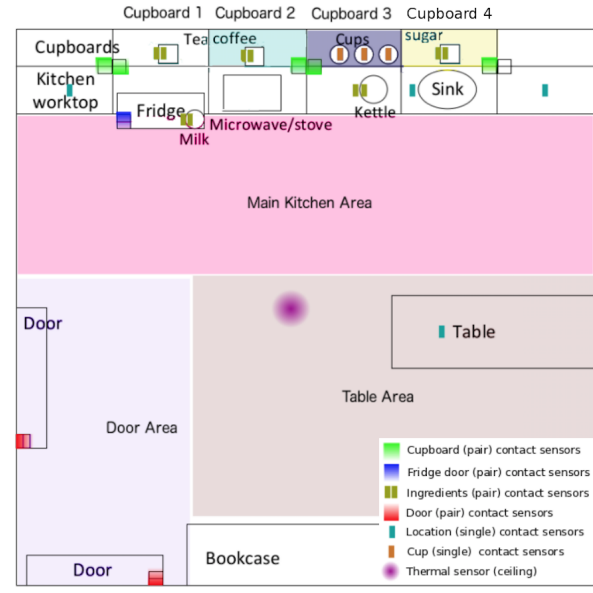

Fig. 1: Smart kitchen layout at SERG.

Data was collected from three types of sensors: (i) contact sensors, (ii) thermal sensors, and (iii) accelerometers. The contact sensors were attached to objects with which the user has interaction in the kitchen in the context of the ADLs considered in this paper: doors, cupboards, refrigerator, cups, containers (tea, coffee, sugar and milk), and location areas (kitchen worktop, table and sink). The contact sensors combine wireless transmitters and magnetic switches. The signals from the contact sensors have two possible states (on or off) and are monitored and collected by SensorCentral [12], a sensor data platform, for further processing and data analysis. The contact sensors (CS) are represented in Figure 1 as rectangles divided into two parts that can be separated ('on' state) or

\footnotetext{
${ }^{1}$ https://www.ulster.ac.uk/research/institutes/computerscience/groups/smart-environments/about
} 
joint ('off' state). Note the colour codes in the legend that indicate to which objects are attached. The details of the data collection, preprocessing and analysis of the thermal sensors and accelerometers are beyond the scope of this paper and will be addressed comprehensively in future work.

Two thermal sensors (TS) were used: (i) one in the ceiling of the kitchen and (ii) one on the kitchen worktop. The thermal sensor mounted in the ceiling of the kitchen is in a central position where it can identify the main areas in the kitchen: (i) main kitchen area, (ii) table area, and (iii) areas around the doors (refer to Fig. 1). The thermal sensor placed on the kitchen worktop was in a horizontal position and directed towards the table to cover the widest area possible. The thermal sensors have a resolution of $32 \times 31$, a $90^{\circ}$ by $86^{\circ}$ field of view that provides a coverage area of $6 \mathrm{~m}$ by $6 \mathrm{~m}$ at a height of $2.5 \mathrm{~m}$, and a sample rate of $10 \mathrm{~Hz}$. Note that the images captured by the thermal sensors do not have enough resolution to be considered as privacy invasive. Figure 3(a) shows the type of thermal sensor used and Figure 3(b) shows a thermal image of a person sitting. The data from the thermal sensors is also monitored and collected in SensorCentral. The main purposes of using thermal sensors in this sensor system are: (i) to identify (in an object classification sense) participants that interact with objects, (ii) to identify the positions of the participants with respect to the kitchen area, and (iii) to identify objects that emit heat when they are being used, such as kettles or pans. It is envisioned that using thermal sensors to capture images from different perspectives provide more insight on how the ADLs are performed.

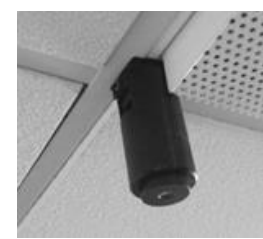

(a)

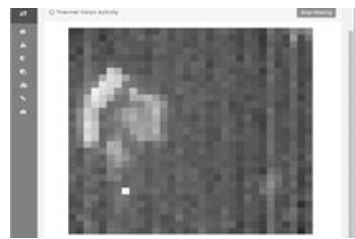

(b)
Fig. 2: (a) Thermal sensor. (b) Example of thermal sensor image.

The accelerometers were the only wearable sensors used and the reason for including them in the ADL environment setup is because they provide more insight into the movements of the users. The accelerometer considered was the Shimmer $2 \mathbf{R}^{2}$. The accelerometer was calibrated using the manufacturer's software prior to the data collection. The accelerometer was placed on the dorsal aspect of the dominant wrist of the participants because that is where wearable devices are usually placed. Data was recorded at a sample rate of $51.2 \mathrm{~Hz}$ with a sensitivity range of $\pm 1.5 \mathrm{G}$. The Shimmer Connect software was used to stream the participants' data through Bluetooth and was then saved in a CSV file.

\footnotetext{
${ }^{2}$ http://www.shimmersensing.com/about/
}

\section{Volunteers And ACTIVIties OF DAily LiVing}

Having explained the rationale behind the way that the sensors were set-up in the smart kitchen in Section III, it is now described how the sample of volunteers was selected and also what type of the Activities of Daily Living (ADLs) were considered in this work.

\section{A. Recruiting Volunteers}

It is worth noting that thirty volunteers were recruited for this project following some specific selection requirements. These requirements were related to their age, gender, and health. Thus, for the creation of the sample, the project worked with volunteers that were from 18 to 45 years old. This agegroup was selected because the majority of the people at these ages are usually healthy and can also exhibit a behaviour that could potentially reveal progressive or temporary health issues. Also, for the experiments conducted, both genders were considered, as the behaviour exhibited usually differs, which could disclose useful information about the way the ADLs are executed and also the time they last. The aim here was to collect information about these two factors, which could indicate abnormal behavioural patterns that could be linked to medical conditions or hazards. At this point, it is worth pointing out that the purpose of the project was not to examine a specific medical condition, but a sample that contains people who are either healthy or have any medical condition. This could result in useful observations about the behaviour that is displayed in each of these cases.

Following the aforementioned criteria, the generated sample of the 30 participants consists of 16 males and 14 females, the youngest and older of whom are 22 and 43 years old respectively. Now, it is remarkable that 28 (approximately $93 \%$ ) of the volunteers claimed that either they are healthy or that they are not aware of any chronic or not medical condition that they suffer from. On the contrary, the remaining participants reported that they face a chronic medical issue (i.e. heart condition and celiac disease). Finally, it should be mentioned that these two volunteers are a male and a female.

Although the sample of the participants that face a medical problem is quite small, it would be interesting to observe and analyse how these two behave compared to all the others. This will be further discussed in Section $\mathrm{V}$.

\section{B. Defining the Activities of Daily Living}

To investigate the behaviour of the participants, two Activities of Daily Living have been considered, the preparation of coffee and tea respectively. These two activities were chosen due to the fact that they can exhibit multiple or similar occurrences during a typical day.

It should be mentioned that for the execution of these two activities, the volunteers had the initiative with respect to the way that they can prepare their drink and the time that they 
will spend to drink it. Consequently, each participant was able to repeat any actions he/she wanted, but it is assumed that he/she could only use the ingredients and equipment that were available for the activity (i.e. coffee, tea, sugar, milk, cups and kettle). The order in which all these items would be used was exclusively dependent on the participant's will. The only restrictions imposed on the participants regarding the completion of the activity were: i) each participant can prepare only one drink, ii) coffee/yea should be drunk in the table and iii) the cup should be placed in the sink after finishing the drink.

Now, to conduct the experiments in the smart kitchen lab (see Fig. 1), a general scenario was followed by the volunteers to prepare their coffee or tea. In this scenario, each participant had to first enter the kitchen using one of the doors, prepare the drink of his/her preference, sit at the table to drink it, leave the cup in the sink when he/she finishes its drinking and finally exit the kitchen. The reason why this scenario was introduced was firstly for the proper set-up of the sensors in the smart kitchen lab and finally for the more accurate tracing of activity steps while the participants use the kitchen's items.

Note that taking into account the initiative of the volunteers and the number of items used in each of the activities, the construction of all the paths (i.e. different sequences of actions) that can be potentially followed for the successful completion of each activity gives more than hundred different ways for the preparation of each drink. Now, if the repetition of some actions(steps) occur, then this number may increase exponentially. For instance, an initial calculation of the total number of unique sequences of steps/action (i.e. paths) showed that there exist around 120 different ways to perform the coffee or tea activity respectively (including no repetition of actions). Examples of such paths are presented below for each activity:

Cof: Door $1 \rightarrow$ Fridge_Door $\rightarrow$ Milk $\rightarrow$ Cupboard $2($ Coffee $)$ $\rightarrow$ Coffee $\rightarrow$ Cupboard $3($ Cup $) \rightarrow C$ up $\rightarrow$ Cupboard $4(S u-$ gar $\rightarrow$ Sugar $\rightarrow$ kettle $\rightarrow$ Hot_water

Tea: Door $1 \rightarrow$ Cupboard $3($ Cup $) \rightarrow C$ Cup $\rightarrow$ Fridge_door $\rightarrow$ Milk $\rightarrow$ Cupboard $1($ Tea $) \rightarrow$ Tea $\rightarrow$ Cupboard4(Sugar $) \rightarrow$ Sugar $\rightarrow$ kettle $\rightarrow H o t \_w a t e r$

Identifying all these sequences of action could help to the extraction of behavioural patterns that would consist of a certain number of steps/actions or a specific order. But, all these will be extensively discussed in the following section.

\section{AnAlysis And Evaluation of RESUlts}

\section{A. Data Processing and Analysis}

The data collected from the contact sensors attached to the objects with which the users interacted was saved to the Sensor Central platform [12] in JavaScript Object Notation (JSON) format. Data was preprocessed using a script in the R language. The following steps were performed to pre-process the data and convert it to a proper format for data analysis: (i) Downloading contact sensor data: The contact sensor data was accessed manually from Sensor Central in a web browser using a specific URL for each sensor in which the corresponding sensor id was indicated. Each contact sensor's data was then copied and saved into a JSON file, which amongst other information contained the sensor id, event code and timestamp in UNIX format. Note that each file contained the historical data for each sensor starting from when they were first installed.

(ii) Preprocessing contact sensor data: The data from each sensor in JSON file was preprocessed and converted into a CSV file including the following columns: eventCode, sensorUUID, dateAndTime and name. The last column was added to indicate the name of the appliance to which the respective sensor was attached to. The dateAndTime column contained the timestamp in standard date and time format (yyyy-mm-dd HH:MM:SS).

(iii) Aggregating contact sensor data: The contact sensor data was combined and aggregated into a single CSV file according to the date and time in which the data was collected, thus following a sequential order. The columns considered were: dateAndTime, name and eventCode.

(iv) Aggregating contact sensor data by user: Since the start and end times for each user data collection was annotated manually, that information was used to select rows from the CSV file resulted in the previous step for each volunteer. Thus, $30 \mathrm{CSV}$ files were created containing the dateAndTime, name and eventCode columns.

The resulting CSV files showed a sequence of interactions of the participant with the objects, and when and how they were used based on the timestamp and event code respectively. Note that for producing the sunburst diagram of action sequences for each user shown in Fig. ??, the sequence of unique consecutive interactions was considered, in this case without taking into account the temporal aspect. This diagram visualises the number of steps required for each volunteer to complete the coffee or tea activity that was chosen and also presents the repetitions that took place throughout the activity.

Now, for the temporal analysis of those sequences of actions, each activity (i.e. coffee or tea) was broken into four main stages: i) entering to the room, ii) drink preparation, iii) drinking and iv) exiting the room. This approach was used to explicitly examine how each participant behaves in each stage in terms of time. It is noteworthy that the purpose of this temporal analysis is to associate the duration of the subtasks or entire activity with potential abnormal behaviour. To count the time that the volunteers did for each subtask, the CSV files were manually processed based on the timestamps and the event codes of the sensors.

For example, the duration of the entering process can be defined as the time past from the activation of the door sensor until the first reading recorded from any of the object sensor involved in the preparation of the chosen drink. Similarly, the duration of the drink preparation stage can be counted 
by getting as starting point the first sensor reading related to the preparation process (i.e. ingredient or equipment reading) and as ending point a cup sensor reading that is followed by the prolonged use of that cup. Now, the duration of the drinking process is calculated by considering the timestamps of the cup sensor readings (i.e. event codes), which indicate a prolonged use of the cup. This prolonged use is defined by the continuous changes to the value of the event code of the cup sensor from 0 to 1 and vice versa. Finally, the duration of the exiting process is computed by taking the timestamp of the last zero value of the cup sensor event code as the starting point of this stage and the timestamp of the last door sensor reading as the ending one. Note that for the calculation of the duration of all the different activity stages, all the repeated actions have been included. Hence, applying the methodology described above, the duration of both the activities and their stages was calculated for each volunteer, as shown in Table I.

TABLE I: Activities and Stages Duration (in sec.)

\begin{tabular}{||c|c|c|c|c|c||}
\hline Vol. ID & Entering & Preparation & Drinking & Exiting & Tot. Time \\
\hline \hline 1 & 5 & 144 & 670 & 21 & 840 \\
\hline 2 & 44 & 110 & 481 & 21 & 656 \\
\hline$\ldots$ & $\ldots$ & $\ldots$ & $\ldots$ & $\ldots$ & $\ldots$ \\
\hline 29 & 56 & 84 & 356 & 20 & 516 \\
\hline 30 & 8 & 170 & 759 & 4 & 941 \\
\hline Aver. & 35.07 & 150.27 & 577.47 & 16.27 & 779.08 \\
\hline St. Dev. & 19.26 & 43.93 & 240.28 & 8.09 & 231.99 \\
\hline \hline
\end{tabular}

Next, for the temporal analysis of the activities, the average and standard deviation of the durations were calculated to define the average time of execution per stage and the range under which a behaviour can be considered as normal for each stage and activity respectively. In more details, the range given by the average value and the standard deviation (i.e. $\bar{x} \pm \sigma$ ) will denote the durations that correspond to normal behaviour. Thus, any duration that falls outside this range could potentially represent an abnormal behaviour for either a stage of the activity or the entire activity.

Now, to ensure that a normal behaviour will not be accidentally identified as abnormal due to its boundary value (i.e. the duration value is close to the minimum or maximum range values but outside that range), the $1.5 \sigma$ is chosen for the creation of the normal behaviour range $\mathrm{e}^{3}$. This range (i.e. $\bar{x} \pm 1.5 \sigma$ ) in the normal distribution covers the $86.6 \%$ of the sample. Theoretically speaking, the reference to the normal distribution is made because of the fact that the duration of each stage of the activities follows that distribution.

To further analyse the activities considered, the Cumulative Distribution Function (CDF) is used [13]. The CDF will give the probability that an activity/stage duration takes a value that is less or equal to a certain value [8]. Thus, to determine whether a behaviour is abnormal or not, an upper and lower bound duration is defined for each activity and stage (given

\footnotetext{
${ }^{3}$ Note that for the size of the sample, the selection of the $1.5 \sigma$ gives a representative range of values for the duration of normally executed activities.
}

by the $1.5 \sigma$ range). CDF will actually show the probability of getting abnormal behaviours related to the early and late completion of an activity or stage respectively.

\section{B. Evaluating the Outcomes}

Using the duration values of Table I for Cumulative Distribution Function, different graphs were produced for the analysis of tea and coffee activities. Specifically, these graphs show the probability over the duration for each activity and stage.

To examine the probability of getting abnormal behaviours that can be used as an indicator for progressive health issues or hazard, the sample of the 30 volunteers was organised into groups according to their gender, age and activity ${ }^{4}$.

Starting with the overall duration of the activities (see graphs in Fig. 3), it is observed for example in the coffee activity that only two participants exhibit behaviour that can resemble an abnormal behaviour as the total time required for the completion of their activity is either considerably low or high. As is reasonable, these two values lie outside the $1.5 \sigma$ (i.e. $86.6 \%$ ) that is considered as the spectrum with all the normal behaviours $^{5}$. In general, this means that the probability of an abnormal behaviour related to the execution time of an activity (resp. stage) is equal to $0.134(13.4 \%)$, which corresponds to four participants that could potentially present an abnormal behaviour for the size of this sample (i.e 30 volunteers).

Now, applying this probability for the sample of volunteers that performed the coffee activity (i.e. 12 volunteers), it is noticed that approximately two of them are expected to have abnormal behaviour (as $13.4 \% \times 12=1.61$ ), which indeed happens as two participants were found to be out of the spectrum of normal behaviour (see Fig.3(a)). Similarly, the tea activity was performed by 18 participants with 3 of them being outside the $1.5 \sigma$ range as shown in the graph of Fig. 3(a). Now, calculating the probability of abnormal behaviour for the sample of the tea activity and translating it into the expected number of such behaviours, this is equal to 2.41 . In this case, the real value is very close to that of the projection indicating the abnormal behaviour with quite good accuracy.

Examining the volunteers' behaviour with respect to their gender, the graph in Fig. 3(b) shows that two male (resp. two female) participants (for both activities) are not in the normal behaviour spectrum defined. Comparing this value with the probability projection for the males (resp. females) ${ }^{6}$, which is equal to 2.14 (resp. 1.88) persons, it can be concluded that the real behaviour can be precisely depicted using the CDF for these subgroups of the main sample. Similary, the probability projection values for the participants of the two age-groups are

\footnotetext{
${ }^{4}$ The different groups for each of these categories are defined as:i) male and female, ii) below and over 30 years old and iii) coffee and tea.

${ }^{5}$ Note that the $1.5 \sigma$ is defined in all the graphs as the range from 0.067 to 0.933 .

${ }^{6}$ Note that the sample consists of 16 males and 14 females, and of 18 and 12 participants under and over 30 years old respectively.
} 

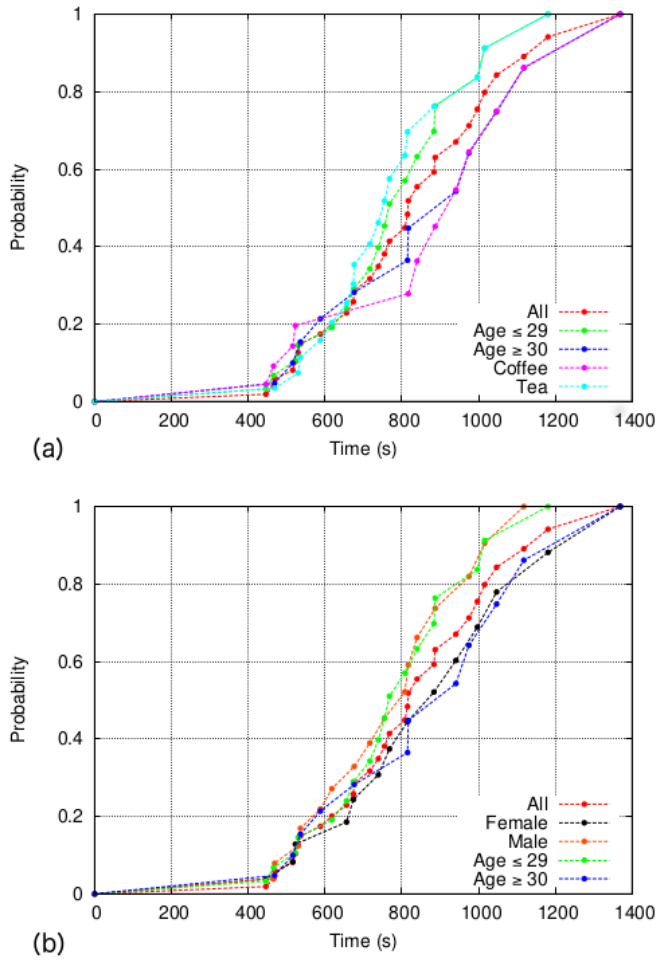

Fig. 3: CDF of Duration for (a) each gender and age-group and (b) each gender and activity performed respectively.

2.41 and 1.61 for the under and over 30 years old respectively. These values are close to the number of participants whose behaviour is considered as abnormal, which is 2 per group.

At this point, the graphs shown in Fig. 4(a) and (b) represent the duration of the entering and exiting stages of the activities respectively. Discussing the CDF results for these two stages, it is noticed that for the entering process of the coffee and tea activities, 4 participants exhibit abnormal behaviour in each case, as shown in Fig. 4(a). Comparing this value to that of the probability for each activity (i.e. 1.61 for coffee and 2.41 for tea), it is observed that the number of abnormal behaviour exhibited in reality is higher than the projection values of these samples. In this case, looking closer at the graph, it can be observed that most occurrences of abnormal behaviour relate to the early completion of the entering stage, which is not necessarily worrying for the health of the participant, but it could be used as an indicator to trigger an alarm. Moreover, regarding the analysis of the results for the age-groups of the entering stage, the probability values are 2.41 and 1.61 for the age-group below and over 30 respectively. Comparing those values with the ones given by the exiting graph, it is observed that 3 persons from the under 30 group present an abnormal behaviour, which is close to the projection value. Contrarily, in the other group, the number of participants that exhibit abnormal behaviour is almost two times the projection value (i.e. 3 volunteers), which results from early completion
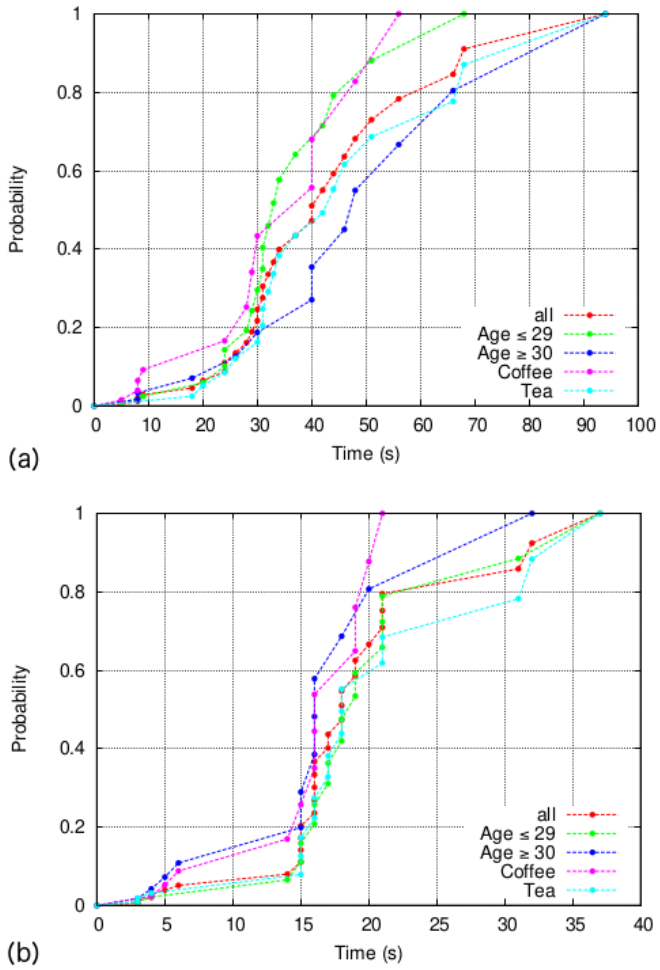

Fig. 4: CDF of (a) Entering and (b) Exiting kitchen for agegroups and activities performed.

of the stage by 2 person ${ }^{7}$.

Following the same approach for the exiting stage (see Fig. 4(b)), the abnormal behaviour projection values are 2.41 and 1.61 for the tea and coffee and below and over 30 age-groups respectively. On the contrary, the values extracted from the respective graph correspond to 4, 3, 2 and 3 participants with abnormal behaviour for the tea, coffee, under 30 years old and over 30 years old respectively. This shows that the only case where the two values are very close is the age-group that is below 30. In all the other cases, there exist a significant difference between the two values. This could result from the fact that in all the cases the volunteers leave the kitchen very quickly, which is not necessarily due to an "abnormal" behaviour that is related to a health issue, but could be because of the fact that the participants hurry. Consequently, from the analysis of both the entering and exiting process, it could be concluded that $\mathrm{CDF}$ cannot give a very accurate projection for the early completion of these phases, but it could give useful information about their late completions that is highly-likely to be linked to a health issue or hazard.

In the last part of the section, it is discussed the probability of having abnormal behaviours during the main stages of the activities, i.e. the preparation and drinking processes. Starting

\footnotetext{
${ }^{7}$ Note that this fact is not necessarily or always related to an abnormal behaviour
} 

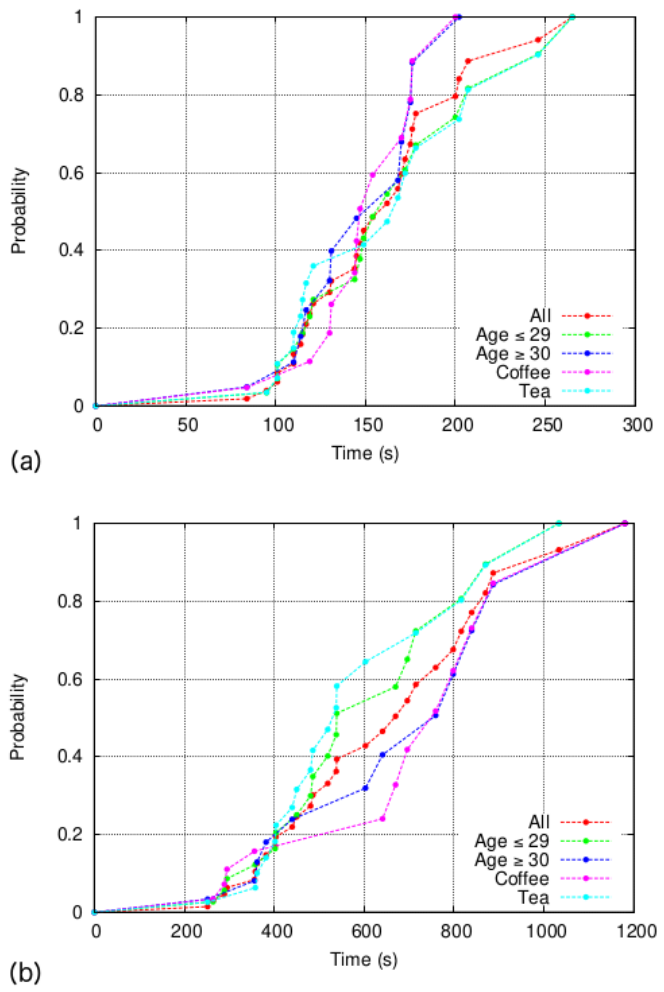

(b)

Fig. 5: CDF of (a) Preparing and (b) Drinking coffee or tea for age-groups.

with the preparation of the drinks, as is noticed from the graph in Fig. 5(a), the number of behaviours that do not belong to the $1.5 \sigma$ is equal to 2 for the coffee and tea respectively, which are close to the values given to the probability for those two samples (i.e. 1.61 for the coffee and 2.41 for the tea). The same observation is made for the two age-groups as the values of the graph show that two participant exhibit abnormal behaviour in each case and also that the values that derive from the probability are 2.41 for the participants under 30 years old and 1.61 for the others. Once again, this indicates that the abnormal behaviour in reality "matches" the one given by the probabilistic analysis in the case of the drink preparation.

Continuing with the drinking process, it is noticed in the graph of Fig. 5(b) that for both age-groups, the number of volunteers that show an abnormal behaviour is equal to 2. Comparing this value to those given by the probability, it can be concluded that the expected abnormal behaviour is observed in the real environment for these samples. Now, for the drinking of the coffee and tea, 2 abnormal behaviours are captured in each activity according to the respective graph, which is also what is shown by the probability values for the coffee and tea activities that are equal to 1.61 and 2.41 respectively.

In general, from the evaluation of the results presented for the sample of the 30 volunteers, it could be concluded that the probabilistic analysis using CDF can provide very accurate results with respect to the presence of abnormal behaviour if the duration of the stages/activities last more than one minute. On the contrary, the results for the short stages of the activities show that the projection of abnormal behaviour given from the CDF is not always precise. Finally, it could be claimed that this analysis model could be used for the training of machine learning algorithms to improve their capability of predicting abnormal behaviour during the execution of the ADLs.

\section{CONCLUSION}

\section{REFERENCES}

[1] O. Aran, D. Sanchez-Cortez, M. Do, and D. Gatica-Perez, "Anomaly detection in elderly daily behavior in ambient sensing environments", International Workshop on Human Behavior Understanding, Springer, pp. 51-67, 2016.

[2] L. Chen, C.D. Nugent, and H. Want, "A Knowledge-Driven Approach to Activity Recognition in Smart Homes", IEEE Transactions on Knowledge and Data Engineering, IEEE, Vol. 24, No. 6, pp. 961-974, 2012.

[3] L. Chen, J. Hoey, C.D. Nugent, D.J. Cook, and Z. Yu, "SensorBased Activity Recognition", IEEE Transactions on Systems, Man, and Cybernetics, Part C (Applications and Reviews), IEEE, Vol. 42, No. 6, pp. 790-808, 2012.

[4] M. Ermes, J. Pärkkä, J. Mäntyjärvi, and I. Korhonen, "Detection of daily activities and sports with wearable sensors in controlled and uncontrolled conditions", IEEE Transactions on Information Technology in Biomedicine, Vol. 12, No. 1, pp. 20-26, 2008.

[5] M. Garcia-Constantino, A. Konios, and C. Nugent. "Modelling Activities of Daily Living with Petri nets", Advanced Technologies for Smarter Assisted Living solutions: Towards an open Smart Home infrastructure (SmarterAAL). 16th IEEE International Conference on Pervasive Computing and Communications (PerCom), pp. 866-871, 2018.

[6] Y. Hong, I. Kim, S. Ahn, and H. Kim. "Activity recognition using wearable sensors for elder care", Second International Conference on Future Generation Communication and Networking, IEEE, vol. 2, pp. 302-305, 2008.

[7] A. Konios, Y. Jing, M. Eastwookd, and B. Tan. "Unifying and Analysing Activities of Daily Living in Extra Care Homes", IEEE 16th International Conference on Pervasive Intelligence and Computing, IEEE, 2018.

[8] E. L. Lehmann and G. Casella, "Theory of Point Estimation", SpringerVerlag, 1998.

[9] A. Lotfi, C. Langensiepen, S.M. Mahmoud, and M.J. Akhlaghinia "Smart homes for the elderly dementia sufferers: identification and prediction of abnormal behaviour", Journal of Ambient Intelligence and Humanized Computing, Springer, Vol. 3, No. 3, pp. 205-218, 2012.

[10] S. Mahmoud, A. Lotfi, and C. Langensiepen. "Behavioural Pattern Identification and Prediction in Intelligent Environments", Applied Soft Computing, Elsevier, Vol. 13, No. 4, pp. 1813-1822, 2013.

[11] L. Meng, C. Miao, and C. Leung. "Towards Online and Personalized Daily Activity Recognition, Habit Modeling, and Anomaly Detection for the Solitary Elderly Through Unobtrusive Sensing", Multimedia Tools and Applications, Springer, Vol. 76, No. 8, 2017.

[12] J. Rafferty, J. Synnott, A. Ennis, C. Nugent, I. McChesney, and I. Cleland. "SensorCentral: A Research Oriented, Device Agnostic, Sensor Data Platform", International Conference on Ubiquitous Computing and Ambient Intelligence, Springer, pp. 97-108, 2017.

[13] N. V., Sarlis and S.-R. G., Christopoulos. "Natural time analysis of the Centennial Earthquake Catalog", Chaos: An Interdisciplinary Journal of Nonlinear Science, Vol. 22, No. 2, pp.23-123, 2012. 
[14] N.K. Suryadevara, A. Gaddam, R.K. Rayudu, and S.C. Mukhopadhyay. "Wireless Sensors Network Based Safe Home to Care Elderly People: Behaviour Detection”, Procedia Engineering, Elsevier, Vol. 25, pp. 9699, 2011.

[15] E. Tapia, S. Intille, and K. Larson, "Activity Recognition in the Home Setting Using Simple and Ubiquitous Sensors", Pervasive Computing, Springer, Vol. 4, pp. 158-175, 2004.

[16] L. Wang, T. Gu, X. Tao, H. Chen, and J. Lu, "Recognizing multi-user activities using wearable sensors in a smart home", Pervasive and Mobile Computing, Elsevier, Vol. 7, No. 3, pp. 287-298, 2011.

[17] T. Zhao, H. Ni, X. Zhou, L. Qiang, D. Zhang, and Z. Yu, "Detecting Abnormal Patterns of Daily Activities for the Elderly Living Alone", International Conference on Health Information Science, Springer, pp. 95-108, 2014. 